\title{
Análise Comparativa de Sistemas de Leitura de Tela no Apoio ao Ensino de Pessoas com Deficiência Visual
}

\author{
Marcos Silva $^{1}$, Bruno Batista ${ }^{1}$, Vitor Amaral ${ }^{1}$, Geisy Anny Venancio ${ }^{1}$, \\ Priscila Fernandes $^{1}$, Vitor Bremgartner ${ }^{1}$ e Luis Rivero ${ }^{2}$ \\ ${ }^{1}$ Instituto Federal de Educação, Ciências e Tecnologia e do Amazonas (IFAM) \\ Manaus, AM - Brasil \\ ${ }^{2}$ Programa de Pós-Graduação em Ciência da Computação (PPGCC), \\ Universidade Federal do Maranhão (UFMA) - São Luís, MA - Brasil \\ \{2021000169, 2020002122, 2021000113, geisy.venancio, priscila.fernandes, \\ vitorbref\}@ifam.edu.br, luisrivero@nca.ufma.br
}

\begin{abstract}
Accessibility technologies help countless people with special needs, such as those with visual impairment, which is a limiting factor, especially for those who have complete loss of sight. In the virtual environment, this type of disability makes it impossible for these users to read a book or take an online course, for example. However, solutions capable of transcribing in audio through screen capture, or reading the data obtained aloud to the user, were created. In this work, these solutions were analyzed in order to identify their characteristics, functionalities and advantages, so that education professionals and/or the students can choose the one that best suits their needs.
\end{abstract}

Resumo. As tecnologias de acessibilidade ajudam inúmeras pessoas com necessidades especiais, como as que possuem deficiência visual, que é um fator limitante, principalmente para aquelas que possuem perda total da visão. No ambiente virtual, este tipo de deficiência impossibilita esses usuários de ler um livro ou realizar um curso online, por exemplo. No entanto, foram criadas soluções capazes de transcrever em áudio através de captura de tela, ou ler os dados obtidos em voz alta para o usuário. No presente trabalho, estas soluções foram analisadas a fim de identificar as características, funcionalidades e vantagens, para que profissionais da educação elou os mesmos estudantes possam escolher a que melhor se adéqua às suas necessidades.

\section{Introdução}

Segundo o IBGE (Instituto Brasileiro de Geografia e Estatística) em 2019, havia 6,5 milhões de pessoas com deficiência visual, sendo aproximadamente 550 mil cegas e 6 milhões com baixa visão [Lemos et al. 2019]. Segundo Leonardo et al. (2009), os resultados obtidos por uma pesquisa entre professores indicam que tanto as escolas públicas como as privadas ainda não possuem infraestrutura adequada para desenvolver projetos inclusivos.

Devido à crise sanitária por motivos da Covid-19, os deficientes visuais tiveram que enfrentar o aprendizado rápido de tecnologias para prosseguir com sua aprendizagem e inclusão social. Portanto, o uso de tecnologias de informação e comunicação pode auxiliar estes usuários no processo de ensino de aprendizagem. Nesse contexto, neste trabalho 
foram analisadas plataformas e aplicativos que têm como principal objetivo a conversão de textos presentes seja na tela ou digitado para voz, facilitando assim a aprendizagem de deficientes visuais que poderiam ouvir livros, revistas, artigos científicos, entre outros. Optou-se pela análise deste tipo de sistemas uma vez que estudos apontam que pessoas com deficiência visual têm audição mais aguçada [Boas et al. 2011].

O seguinte trabalho está organizado como segue, a Seção 2 apresenta a fundamentação teórica e trabalhos relacionados. Já a Seção 3 apresenta a metodologia deste trabalho. Por sua vez, a Seção 4 apresenta os sistemas identificados e uma discussão comparando os resultados. Finalmente, a Seção 5 apresenta as conclusões e perspectivas futuras deste trabalho.

\section{Fundamentação Teórica e Trabalhos Relacionados}

A baixa visão é a perda grave de visão, que não pode ser corrigida por tratamento clínico ou cirúrgico, nem com óculos convencionais [Domingues et al. 2010]. Ela pode ser descrita como qualquer grau de dificuldade que causa incapacidade funcional e diminua o desempenho visual. Além disso, a inclusão educacional é entendida pela matrícula permanente de escolares com necessidades educacionais especiais.

A inclusão social é a modelagem da sociedade para receber em seu meio pessoas portadoras de necessidades especiais, enquanto estas se preparam para exercer seu papel na sociedade [Martini 2005]. Os principais empecilhos encontrado durante o processo de aprendizagem em função socio-educacional, são [Ferroni and Gasparetto 2012]: (a) a ausência de adequação de professores nomeados para esta demanda; (b) a falta de infraestrutura adequada; (c) poucos materiais e recursos; e (d) pouca retenção na aprendizagem cuja visão é obrigatória.

Rabello (2007) executou uma pesquisa na utilização de programas para deficientes visuais, visando a eficácia da capacidade de produção e retenção de conhecimento. Foi utilizado o programa Dosvox e se constatou que a capacidade dos deficientes digitarem e aprenderem se deu por um tempo muito mais rápido depois da utilização do programa. Vale ressaltar que os deficientes foram submetidos a aulas durante alguns meses e feitas as comparações de resultados iniciais e finais.

De acordo com Capellini et al. (2011), a formação do indivíduo deve ser contínua e com parceria com a sociedade, assim melhorando a interação entre os departamentos de educação especial e ensino comum. Além disso, os futuros professores deveriam ser treinados na prática durante sua formação, para melhor atender suas atividades e necessidades do ambiente escolar.

Almeida et al. (2016), afirma que apesar dos celulares de ponta já virem com funcionalidades que ajudam as pessoas cegas, muitas das vezes estas funcionalidades não suprem todas as necessidades do usuário, precisando recorrer a instalação de outros programas auxiliares. Além disso, a falta dos mesmos pode fazer com que os deficientes fiquem sem conseguir utilizar o aparelho $100 \%$.

\section{Metodologia}

Visto que muitas pessoas enfrentam problemas visuais, surgiu a necessidade de analisar se os programas e aplicativos disponíveis para auxilia-los suprem todas as suas necessidades básicas. Considerando a pandemia da COVID-19, a utilização da internet se tornou 
importante no processo de formação no ensino superior. Uma vez que a informática está ficando cada vez mais presente na vida das pessoas. Desta forma, ter softwares que auxiliam os deficientes visuais irá ajudar não só na sua educação mas também na inclusão social.

Neste trabalho foi utilizada a técnica de becnhmarking [Martins et al. 2010]. O benchmarking pode ser definido como um processo contínuo e sistemático para avaliar produtos, serviços e processo de trabalho de organizações que são reconhecidas como representantes das melhores práticas, com a finalidade de melhoria organizacional. Neste trabalho, esta técnica foi utilizada para fazer uma análise comparativa de sistemas de leitura de tela, considerando as sugestões da sua aplicação na engenharia de software [Vazquez and Simões 2016].

A coleta de dados se deu por meio de escolha de critérios para definir quais são os programas do gênero que serão o objeto de comparação. Foram considerados artigos científicos no Google Acadêmico e blogs, e a análise de resumos disponibilizados pelas fabricantes das plataformas nas lojas de aplicativos. Após esta etapa, foi feita a análise dos programas por meio da utilização e por meio das próprias informações prestadas pelo fabricante, seja por informações gerais ou informações específicas como: funcionalidades, vantagens e desvantagens e entre outros. Finalmente, foi feito um resumo textual de cada um dos programas para se ter uma noção básica do que cada um poderia fazer e proporcionar. Foram observadas as características e funcionalidades de cada programa e anotadas em duas tabelas principais sendo uma para aplicações em desktop e outra para aplicativos móveis. Finalmente, foi feito o comparativo das funções mais relevantes de cada sistema analisado, mostrando a capacidade que cada programa exercia naquela função.

\section{Resultados Alcançados}

Os aplicativos foram selecionados de acordo com as avaliações, considerando os mais indicados em lojas de aplicativos que: (a) funcionassem de forma a abranger vários sistemas operacionais; (b) possuíssem acessibilidade em relação a recursos financeiros (plataformas não pagas); (c) possuíssem acessibilidade de documentos feitos pelos próprios desenvolvedores das plataformas; (d) tivessem quantidade de downloads acima de 1000; e tivessem comentários positivos que favoreceram a escolha dessas plataformas.

A pesquisa é exploratória uma vez que se deseja conhecer, analisar e verificar os ambientes mediáticos. Os sistemas selecionados foram: Dosvox, Jaws, Orca, NVDA, Texto para voz-leia em voz alta, TTS reader-lê em voz alta todos os livros!, Talkback, VoiceOver, Dina e CPqD Alcance+.

\subsection{Sistemas Identificados}

As plataformas escolhidas para auxiliar os deficientes por meio de conversão de texto em voz além de outras funcionalidades serão detalhadas a seguir:

Dosvox [Borges 1998]: O DOSVOX é um sistema para microcomputadores da linha PC que se comunica com o usuário através de síntese de voz, viabilizando, o uso de computadores por deficientes visuais[BORGES 2016]. Além disso, este é um programa que auxilia o usuário com um sistema de voz, desenvolvido este pelo instituto Tércio Pacite relacionado à Universidade Federal do Rio de Janeiro (UFRJ). O sistema tem por finalidade ajudar deficientes visuais de qualquer tipo a manusearem computadores e suas 
funcionalidades básicas. Uma diferença notada deste programa de acordo com o próprio instituto Tércio Pacite é que ele foi desenvolvido pensando como principal ponto o fato de que o programa será utilizado por deficientes visuais.

Jaws [Sonza and Santarosa 2003]: É um programa desenvolvido pela empresa norte-americana Henter-Joyce pertencente ao grupo Freedom Scientific. O Jaws para Windows é um leitor de telas que permite facilmente o acesso ao computador a pessoas cegas ou amblíopes. Com o Jaws, qualquer usuário deficiente visual pode trabalhar tão ou mais rapidamente do que uma pessoa que veja normalmente, utilizando teclas de atalho. Estima-se que atualmente a quantidade de usuários deste programa esteja em torno de 50.000, espalhados por vários países. É um software de fácil utilização, eficiente e a velocidade pode ser ajustável conforme o nível de cada usuário.Este programa foi desenvolvido para ser utilizado em sistema operacional Windows, funcionando em todas as versões, a partir do Windows Vista.

Orca [Bal et al. 1992]: é um aplicativo leitor de tela que tem como função principal comutar imagens gráficas em voz assim como texto. Ele é escrito em linguagem Python e usa uma biblioteca GTK2. É licenciado pela licença LGPL e, portanto, o usuário pode desenvolver com o GTK software aberto, livre ou mesmo comercial e não-livre, sem ter de gastar nada com licenças.

NVDA [Pansanato and Silva 2017]: permite que pessoas cegas e deficientes visuais acessem e interajam com o sistema operacional Windows e muitos aplicativos de terceiros. O sistema é gratuito para uso global, para que o usuário não se preocupe com o custo do software de leitura de tela. Rápido, leve e compacto garante estabilidade superior, confiabilidade e menos acidentes, para que o usuário seja mais produtivo, a plataforma está disponível em vários idiomas. Ótimo se o usuário fala um idioma minoritário, onde as opções são limitadas ou caras.

Texto para voz- leia em voz alta (mobile): É uma plataforma que usa diversos tipos de entrada de texto para um texto em voz. O usuário pode carregar texto de arquivos (PDF e TXT) como seu e-book, jornais, reportagem e e-mail; pode inserir ou digitar texto facilmente através do reconhecimento de fala do google; pode até digitalizar texto de livros ou documentos reais via câmera OCR (apenas caracteres latinos são suportados); e pode carregar o texto de seus sites favoritos (por exemplo, HTML e leitor de páginas da web).

TTS Reader - lê em voz alta todos os livros (mobile): Lançado em 15 de janeiro de 2019, seu desenvolvedor Librera diz que: "é um leitor de livros, leitor TTS, leitor alto, leitor de voz, tudo em um, leia qualquer formato de livro em voz alta, usando o mecanismo TTS de sua escolha-EPUB, MOBI, TXT, FB2, PDF, DJVU, RTF, AZW, HTML, RTF, ODT e até mesmo páginas da web. TTS Reader é um aplicativo avançado, altamente personalizável e poderoso baseado no Librera Book Reader Professional". Ele está disponível em vários sistemas operacionais (exceto em IOS). No computador ele só funciona com o auxílio de um emulador, mas não sendo possível ler a tela completa do computador e sim a tela do próprio emulador, porém do mesmo modo o usuário deveria usar o mouse para manusear o emulador, logo é mais vantajoso usar um programa para desktop.

Talkback (mobile): É um aplicativo que faz parte do pacote de acessibilidade do android, e tem como finalidade ajudar usuários cegos e deficientes visuais a usar seus dis- 
positivos móveis. Essa ferramenta possibilita o usuário explorar seu dispositivo, através do deslize do dedo sobre a tela, descrevendo em voz alta tudo que se toca, como aplicativos, mensagens de texto, configurações, status da bateria, do sinal de celular e wifi. Sendo possível selecionar o item apenas com dois toques, é possível abrir outros aplicativos como spotify, tocar play list de músicas, ler mensagens no whatsapp, navegar na internet, alteração de idiomas, ler a tela completa do dispositivo, é acessível somente para mobile.

$\mathrm{CPqD}$ Alcance+ (mobile): é um aplicativo brasileiro de laucher disponível na plataforma android, que tem como função auxiliar o usuário com deficiência visual a utilizar seu dispositivo móvel de forma autônoma, através da leitura de tela. Ao instalar o aplicativo a interface do dispositivo é alterada e dividida em duas grandes grades padronizadas, e ao deslizar o dedo pela tela, a ferramenta ler em voz alta a opção escolhida e realiza inúmeras funções como: além de realizar a leitura da tela, este altera a interface do dispositivo, é gratuito, realizar ligações telefônicas, acessar a lista de contatos, e-mails, data e hora, porcentagem da bateria, despertador com lembrete de voz, reprodutor de música, previsão do tempo, leitor de arquivos de texto, acesso a internet e modificações de aparência como fonte, voz e nível de experiência do usuário.

Dina (mobile): Lançado em 12 de novembro de 2020, Dina é um aplicativo que reconhece objetos, cores e textos utilizando sua câmera e os fala em voz alta. Ele foi projetado para ajudar deficientes visuais, oferecendo os seguintes recursos: funciona offline, sem necessidade de conta, sem custo, sem anúncios e sem limites de uso e funcionalidade.

VoiceOver (mobile): É um sistema do fabricante Apple. É um leitor de tela baseado em gestos. $\mathrm{O}$ usuário pode usar o iPhone mesmo que o usuário não possa ver a tela. O VoiceOver fornece descrições audíveis do que está na tela, desde o nível da bateria até quem está ligando e em qual app o dedo do usuário está. O usuário também pode ajustar a velocidade da fala e o tom de voz conforme as suas necessidades, ao acessar uma nova tela, o VoiceOver reproduz um som, e seleciona e fala o primeiro item na tela (normalmente no canto superior esquerdo). O VoiceOver fala quando a tela muda da orientação horizontal para a vertical, quando a tela escurece ou é bloqueada e o que está ativado na Tela Bloqueada ao despertar o iPhone.

\subsection{Análise Comparativa}

As Tabelas 1 e 2 contém os resultados de comparação entre as plataformas. A seguir, estas funcionalidades serão discutidas.

Com relação à função de Síntese de Fala, esta se apresenta em todos os programas listados. Ela é o processo de produção artificial de fala humana e pode ser implementado em software ou hardware. Um sistema texto-fala converte texto ortográfico em fala. Outros sistemas interpretam representação linguística simbólica (como transcrição fonética) em fala, ou seja, em todos os casos traduz em voz alta aquilo que o usuário está incapacitado de enxergar na tela, porém tem a disponibilidade de ouvir. Com destaque para VoiceOver e NVDA que através das configurações podem alterar a velocidade e o tom da voz de leitura para os iniciantes não se perderem. Dosvox possibilita a busca personalizada em Janela. Finalmente, o Texto para Voz - Leia em voz alta e Talkback possuem temporizador de Voz para configurar o timer que vai finalizar a narração do livro.

A Disponibilidade de Idioma é um dos maiores avanços quando o assunto é aces- 
Tabela 1. Análise comparativa dos sistemas desktop

\begin{tabular}{|c|c|c|c|c|c|}
\hline Programas / Funções & 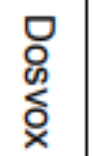 & $\sum_{\infty}^{c}$ & 옳 & $\underset{\delta}{z}$ & @ৃ \\
\hline Sistema de síntese de fala. & AT & AT & AP & AT & AT \\
\hline Disponibilidade de idiomas (fala). & AP & AT & AP & AT & AT \\
\hline Leitor/editor para plataformas diferentes de texto. & AP & AP & $\mathrm{AP}$ & AT & AT \\
\hline Disponibilidade de acesso a internet & AP & AT & $\mathrm{AP}$ & AT & AT \\
\hline Disponibilidade de leitura de texto físico. & $\mathrm{N}$ & $\mathrm{N}$ & $\mathrm{N}$ & $\mathrm{N}$ & AT \\
\hline Personalização vocal da leitura textual. & AT & $\mathrm{AP}$ & AT & AT & AT \\
\hline
\end{tabular}

Legenda: AT-atende totalmente; AP-atende parcialmente; N- não atende.

sibilidade. Alcançar o máximo de usuários com as mais diversas línguas é quase um objetivo comum para todo Software bem-sucedido. Nesse contexto, o Dosvox não está limitado ao Português e se aplica também ao inglês, apesar de ter sido desenvolvido no Rio de Janeiro. Já o NVDA possui a narração do conteúdo na tela e leitura de arquivos para mais de 50 idiomas, proporcionando acesso e principalmente comunicação quase que em qualquer lugar do mundo. Por sua vez, o Jaws tem disponibilidade para mais de 30 idiomas. Dina, CPqd Alcane+ e Texto para voz - Leia em voz alta, não têm disponibilidade para outros idiomas, sendo somente para português.

Uma das formas mais comum de estudo individual e grupal nos dias de hoje se resume à busca de material na internet sobre os assuntos. Para isso os programas de auxílio aos deficientes visuais proporcionam a leitura de arquivos de texto, e alguns ainda permitem a edição e criação do próprio documento, como o Dosvox. Os demais sistemas abrem possibilidades de leitura para arquivos PDF, Microsoft Word (.Doc, .Dox, etc.), sites, TXT, EPUB, FB2, zip etc. Com o leque pode-se então ter acesso a muito conteúdo para estudo de forma independente.

Com relação à disponibilidade da Internet, uma das principais funcionalidades está relacionada à comunicação à distância em tempo real. Quanto a essa funcionalidade, todos os sistemas possuem alguma especificação que os difere. Por exemplo, alguns Vinculam a conexão com lista de Navegadores e comunicação formal e informal através de E-mails e Websites. O NVDA se destaca ao trazer um software de bate-papo na Internet e reprodutores de música.

Com relação à disponibilidade dos sistemas analisados por sistema operacional, o Orca é requisito de instalação no Sistema Linux e outros sistemas baseados em Unix. Já os aplicativos móveis podem ser instalados nos celulares, sendo de acesso mais fácil aos cidadãos como um todo para as versões Android e IOS.

Com relação à disponibilidade de leitura de texto físico, alguns sistemas permitem abrir a câmera para permitir essa leitura. Esta é uma função rara entre as plataformas tanto para desktop ou mobile. Nesse contexto, as plataformas Dina e VoiceOver foram as que se destacaram por disponibilizar essa funcionalidade. 
Tabela 2. Análise comparativa dos sistemas para dispositivos móveis

\begin{tabular}{|c|c|c|c|c|c|}
\hline Programas / Funções & 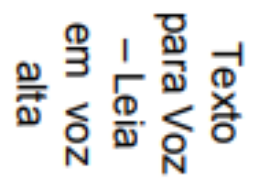 & 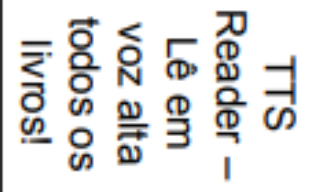 & 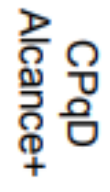 & $\stackrel{\mathrm{g}}{\mathrm{g}}$ & 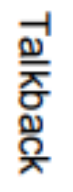 \\
\hline Sistema de síntese de fala. & AT & AT & AT & AT & AT \\
\hline $\begin{array}{l}\text { Disponibilidade de idiomas } \\
\text { (fala). }\end{array}$ & $\mathrm{N}$ & AP & $\mathrm{N}$ & $\mathrm{N}$ & AT \\
\hline $\begin{array}{l}\text { Leitor/editor para } \\
\text { plataformas diferentes de } \\
\text { texto. }\end{array}$ & AT & AT & AT & AP & AT \\
\hline $\begin{array}{l}\text { Disponibilidade de acesso a } \\
\text { internet. }\end{array}$ & $\mathrm{N}$ & AP & AP & AT & AT \\
\hline $\begin{array}{l}\text { Disponibilidade de leitura de } \\
\text { texto físico. }\end{array}$ & $\mathrm{N}$ & $\mathrm{N}$ & AP & AT & $\mathrm{N}$ \\
\hline $\begin{array}{l}\text { Personalização vocal da } \\
\text { leitura textual. }\end{array}$ & AT & AT & AT & $\mathrm{N}$ & AT \\
\hline
\end{tabular}

Legenda: AT-atende totalmente; AP-atende parcialmente; N- não atende.

Finalmente, a personalização vocal de leitura textual é a função de modificar a voz do programa que está comutando o texto para voz, seja voz de homem ou mulher, rápida ou lenta, mais grave ou aguda. Esta função que permite ao usuário ficar mais confortável, já que o mesmo vai ficar muito tempo ouvindo um livro por exemplo. Vale ressaltar que a plataforma Dina foi a única que não disponibilizou essa função, e o Jaws não totalmente.

\section{Conclusões e Trabalhos Futuros}

Considerando as informações reunidas sobre os sistemas de apoio, de todos as plataformas comparadas, o VoiceOver foi o mais completo, apresentando nele todas as funções analisadas. Entretanto ela só está disponível em dispositivos fabricados pela Apple, o que implica que para ter acesso a esta plataforma é necessário comprar dispositivos (IOS) que estão num preço acima do mercado. Desta forma, surge a necessidade de um aplicativo que supra as necessidades dos usuários, mas que tenha um custo de aquisição mais baixo. Outra opção pode ser disponibilizar o VoiceOver para outras plataformas.

De acordo com análise de usuários nas lojas de aplicativos, muitos usuários estão enfrentando problemas de compatibilidade tendo em vista a variação de dispositivos. Alguns dos problemas relatados são: o aplicativo não está apresentando a voz na hora da leitura, o aplicativo não está abrindo, mostrando assim a falta de compatibilidade.

Algumas funções que ficaram de fora da análise comparativa entre os programas foi a disponibilidade de cursos, tutoriais, ou modo de usar as plataformas, visto que os usuários, na maioria das vezes, precisa de auxílio para aprender a usa-las. Deste modo, foi percebido que os programas para desktop apresentavam um manual, mas eles estão disponíveis somente em texto e não em áudio, o que os torna difícil para um usuário acessar. Já os aplicativos mobile não apresentam essas informações e sim um resumo de funções que os aplicativos apresentam, mas não como usar essas funções. 
Após a análise realizada, os autores deste trabalho recomendam o VoiceOver e sugere-se que este seja disponibilizado em outras plataformas e dispositivos. Como trabalho futuro, pretende-se utilizar uma metodologia mais robusta, com pontuação, oriunda da avaliação de usuários, de modo que seja possível realizar um comparativo com base nas opiniões dos usuários e obter indícios dos impactos das ferramentas, na comunidade com deficiência visual.

\section{Agradecimentos}

Parte dos resultados apresentados neste trabalho foram obtidos através do PROJETO ARANOUÁ, financiado pela Samsung Eletrônica da Amazônia Ltda., com recursos previstos na Lei Federal n8.387/1991.

\section{Referências}

Almeida, R., Mesquita, L., Carvalho, R. M., Junior, B., and Andrade, R. (2016). Quando a tecnologia apoia a mobilidade urbana: Uma avaliação sobre a experiência do usuário com aplicações móveis. In Proceedings of the XV Brazilian Symposium on Human Factors in Computer Systems (IHC 2016). Brazilian Society of Computation-SBC, Porto Alegre, Brazil.

Bal, H. E., Kaashoek, M. F., and Tanenbaum, A. S. (1992). Orca: A language for parallel programming of distributed systems. IEEE transactions on software engineering, 18(March):190-205.

Boas, L. V., Muniz, L., Caldas Neto, S. d. S., and Gouveia, M. d. C. L. (2011). Desempenho do processamento auditivo temporal em uma população de cegos. Brazilian Journal of Otorhinolaryngology, 77:504-509.

Borges, J. A. (1998). Dosvox: uma nova realidade educacional para deficientes visuais. Revista Benjamim Constant, 3:24-29.

BORGES, J. A. (2016). Manual do dosvox. Núcleo de Computação Eletrônica da UFRJUniversidade Federal do Rio de Janeiro.; http://www. nce. ufrj. br.

Capellini, V. L. M. F., Rodrigues, O. M. P. R., do Valle, T. G., Melchiori, L. E., Zanata, E. M., Leite, L. P., and Lepre, R. M. (2011). Perfil e avaliação dos participantes de um curso de formação continuada em práticas educacionais inclusivas na modalidade a distância. Práxis Educativa (Brasil), 6(1):79-89.

Domingues, C. d. A., Sá, E. D. d., Carvalho, S. H. R. d., Arruda, S. M. C. d. P., and Simão, V. S. (2010). A educação especial na perspectiva da inclusão escolar. os alunos com deficiência visual: baixa visão e cegueira.

Ferroni, M. C. C. and Gasparetto, M. E. R. F. (2012). Escolares com baixa visão: percepção sobre as dificuldades visuais, opinião sobre as relações com comunidade escolar e o uso de recursos de tecnologia assistiva nas atividades cotidianas. Revista Brasileira de Educação Especial, 18:301-318.

Lemos, G. d. J. M., de Oliveira, M. E. S., and Ferreira, J. N. (2019). Acessibilidade de pessoas com deficiência visual nos serviços de saúde. Revista Científica de Gestão Hospitalar, 1(1):108-112. 
Leonardo, N. S. T., Bray, C. T., and Rossato, S. P. M. (2009). Inclusão escolar: um estudo acerca da implantação da proposta em escolas de ensino básico. Revista Brasileira de Educação Especial, 15:289-306.

Martini, R. (2005). Inclusão digital \& inclusão social. Inclusão social, 1(1).

Martins, S. G., Santos, A. S. d., and Carvalho, L. M. (2010). O benchmarking e sua aplicabilidade em unidades de informação: uma abordagem reflexiva.

Pansanato, L. T. E. and Silva, D. G. D. (2017). Inclusão social de pessoas cegas: treinamento no uso de leitor de tela.

Rabello, S. et al. (2007). O uso do computador no desempenho de atividades de leitura e escrita do escolar com deficiência visual.

Sonza, A. P. and Santarosa, L. M. C. (2003). Ambientes digitais virtuais: acessibilidade aos deficientes visuais. RENOTE, 1(1).

Vazquez, C. E. and Simões, G. S. (2016). Engenharia de Requisitos: software orientado ao negócio. Brasport.

\section{Links para acesso dos sistemas identificados:}

Loja de aplicativos Play Store para sistemas operacionais mobile. Disponível em https://play.google.com/store/apps/details?id=tts.readerhl=ptBRgl = US.Acessoem : 25 de junho de 2021.

Loja de aplicativos Play Store para sistemas operacionais mobile. Disponível em https://play.google.com/store/apps/details?id=com.bluefish.text2voicehl=ptBRgl = US.Acessoem : 25 de junho de 2021.

Loja de aplicativos Play Store para sistemas operacionais mobile. Disponível em https://play.google.com/store/apps/details?id=com.google.android.marvin.talkback. Acesso em: 03 de julho de 2021.

Nv Access- Empowering lives though non visual access to technology. Dispon'1vel em https://www.nvaccess.org/about-nvda/. Acesso em: 24 de junho de 2021.

Gnome help-Comandos da orca. Disponível em https://help.gnome.org/users/orca/stable/commands.html. Acesso em: 24 de junho de 2021.

Imasters- powered by: Totvs developers/Huawei cloud. Disponível em https://imasters.com.br/acessibilidade/conheca-o-adriane-um-gerenciador-e-leitordetela-para-deficientes-visuais. Acesso em: 25 de junho de 2021.

Obs software- desenvolvedor e comercializador de software. Disponível em http://www.osbsoftware.com.br/produto/jaws. Acesso em: 25 de junho de 2021. 